\title{
Fabrication Process of Single CuO Nanowire Devices
}

\author{
Vu Xuan Hien, Kwang-Min Jo, Se-Yun Kim, Joon-Hyung Lee, Jeong-Joo Kim, and Young-Woo Heo*
}

School of Materials Science and Engineering, Kyungpook National University, Daegu 702-701

(Received May 27, 2014, Revised May 29, 2014, Accepted May 30, 2014)

One-dimensional nanostructures such as nanowires have been extensively investigated as a promising type of material for applications of nanoscale technology. The fabrication of single-nanowire devices are consequently important and interesting. This study introduced a feasible method for growing $\mathrm{CuO}$ nanowires on $\mathrm{Cu}$ foils. The nanowires had diameters of $10 \sim 150 \mathrm{~nm}$ and lengths of more than $7 \mu \mathrm{m}$ and were grown by means of thermal oxidation in a vacuum. They were entirely and uniformly grown over the $\mathrm{Cu}$ foil surfaces and could be extracted and dispersed in an ethanol solution for further purposes. In addition, a simple fabrication method for realizing device functionality from a single $\mathrm{CuO}$ nanowire was reported. Fabricated devices were carefully checked by field-emission scanning electron microscopy (SEM). The probability of the realization of a single-CuO-nanowire device relative to that of all other types was estimated to be around $25 \%$. Finally, the I-V characteristics of the devices were analyzed.

Keywords : Nanowire FET, CuO, Growth, Semiconductor device

\section{Introduction}

Single-nanowire-based devices have been widely studied nowadays to investigate the natural properties of materials in the one-dimensional (1D) nanoscale structures. Many studies have been carried out to assess the use of these devices for energy storage, detector and low-power thin-film transistor (TFT) applications [1-4], etc Up to this moment, there are two typical approaches to fabricate the devices. For the first method, nanowires are dispersed in an aqueous solution (typically ethanol) and a droplet of the solution is transferred to an electrode deposited substrate by a coating technique. Although this method is very simple to process, the contact between the wire and the electrode is frequently contaminated by many factors, including contamination of the aqueous solution by non-nanowire species. The second approach is based on a photolithography process in which nanowires are initially transferred onto a substrate, after which electrodes are patterned and deposited onto the wires by photolithography. When using this method, single-nanowire devices can be fabricated by modulating the transfer process.

In recent years, cupric oxide $(\mathrm{CuO})$, a narrow- bandgap metal oxide semiconductor, has attracted much attention. Nanometer-scale $\mathrm{CuO}$ is a material which has potential for use in heterogeneous catalyst [5], photocatalyst [6], gas-sensing [7], and bio-sensing [8] applications. It can also serve as a cathode material in lithium ion batteries [9] and as a photoconductive material [10]. Numerous

* [E-mail] ywheo@knu.ac.kr 
nanostructures of $\mathrm{CuO}$, e.g., nanoparticles [8], nanowires [4], nanobelts [11] and nanoflowers [12], among others, have been synthesized via many physical as well as chemical methods. Interestingly, $\mathrm{CuO}$ nanowires can be grown easily by oxidizing $\mathrm{Cu}$ foil at elevated temperatures $[4,13]$. Thus far, the thermal oxidation of $\mathrm{Cu}$ foil in the presence of oxygen appears to be the most effective route to obtain high-quality $\mathrm{CuO}$ nanowires.

In this paper, $\mathrm{CuO}$ nanowires were synthesized by thermal oxidation method. In addition, a simple fabrication method of a single $\mathrm{CuO}$ nanowire field-effect transistor (FET) was reported.

\section{Experimental Section}

For the $\mathrm{CuO}$ growth, a $1 \times 1 \mathrm{~cm}^{2} \mathrm{Cu}$ foil sample (purity: 99.9\% Cu, $100 \mu \mathrm{m}$ thickness) was cleaned sequentially in a solution of HF:DI-water (1:2), DI water, and ethanol for 3 minutes each before drying with compressed $\mathrm{N}_{2}$. The foil was then fixed at the center of a CVD system. During the treatment, the temperature and pressure were controlled at $450^{\circ} \mathrm{C}$ and 0.1 Torr, respectively. A mixture of oxygen and nitrogen with oxygen partial pressure $\left(\mathrm{O}_{\mathrm{pp}}\right)$ levels of $10 \%, 20 \%$ and $50 \%$ was subsequently introduced for the treatment. The total gas flow rate was $50 \mathrm{sccm}$. After a 12-h treatment, the samples were cooled naturally. The shapes and structures of the wires were characterized by field-emission scanning electron microscopy (FESEM, JSM-6701F). In addition, the nanowires were dispersed on a molybdenum grid for transmission electron microscopy (TEM-Tecnai G2F20 S-TWIN, Philips) observations.

The fabrication steps of the bottom-gate structure field-effect transistor using a single nanowire as a channel are illustrated in Fig. 1. First, the black crust on the $\mathrm{Cu}$ foil surface was scratched and dispersed in $10 \mathrm{ml}$ ethanol. The solution was then treated in an ultrasonic bath for $10 \mathrm{~min}$. After holding it for $24 \mathrm{~h}, 1 \mathrm{ml}$ of the as-dispersed solution was transferred to another $10 \mathrm{ml}$ of ethanol and the new solution was sonicated for $10 \mathrm{~min}$.

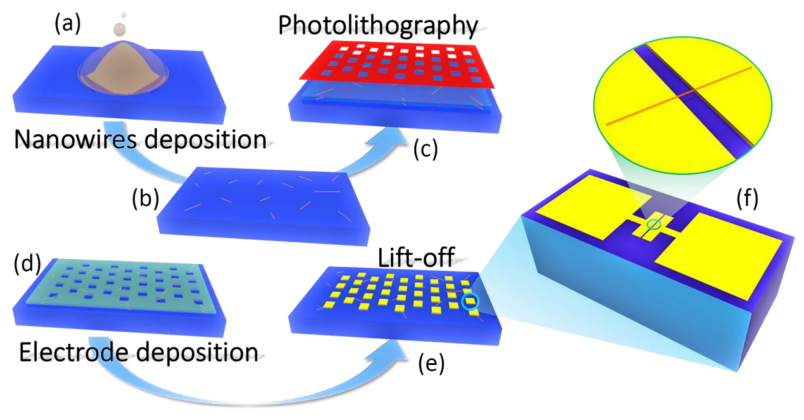

Figure 1. Fabrication steps of the single nanowire device.

Second, the final solution was dropped and spin-coated onto a $2 \times 2 \mathrm{~cm}^{2}$ p-type silicon substrate (a 100-nm $\mathrm{SiO}_{2}$ layer was created on the $\mathrm{Si}$ substrate by thermal oxidation, which was employed as a gate dielectric oxide) at a speed of $1000 \mathrm{rpm}$ for $2 \mathrm{~min}$ (Fig. 1(a, b)). The substrate was subsequently sonicated in an ethanol solution for $30 \mathrm{~min}$ to remove the bulk $\mathrm{CuO}$ and contaminates. Third, bi-layer photoresist (PR) / lift-off resist (LOR) photolithography was used to patterned the electrodes (Fig. 1(c, d)). The substrate was then exposed to oxygen plasma for $1 \mathrm{~min}$ to remove any residual PR on the nanowires. After depositing $\mathrm{Ni} / \mathrm{Au}(40 / 60 \mathrm{~nm})$ for a source/drain electrode by e-beam deposition, the bi-layer was lifted off by Remover PG (Fig. 1e). All devices were then checked by an optical microscope and by SEM. The good single nanowire devices (Fig. 1f) were marked for further measurements. The current-voltage (I-V) characteristics were assessed in the dark at room temperature by an Agilent 4156C semiconductor parameter analyzer.

\section{Results and Discussion}

\section{Synthesis of $\mathrm{CuO}$ nanowires}

The influence of the oxygen partial pressure on the growth of $\mathrm{CuO}$ nanowires is shown in Fig. 2. This figure clearly shows that the nanowires are denser and longer under a higher oxygen partial pressure. The diameters and lengths of the observed wires were approximately 


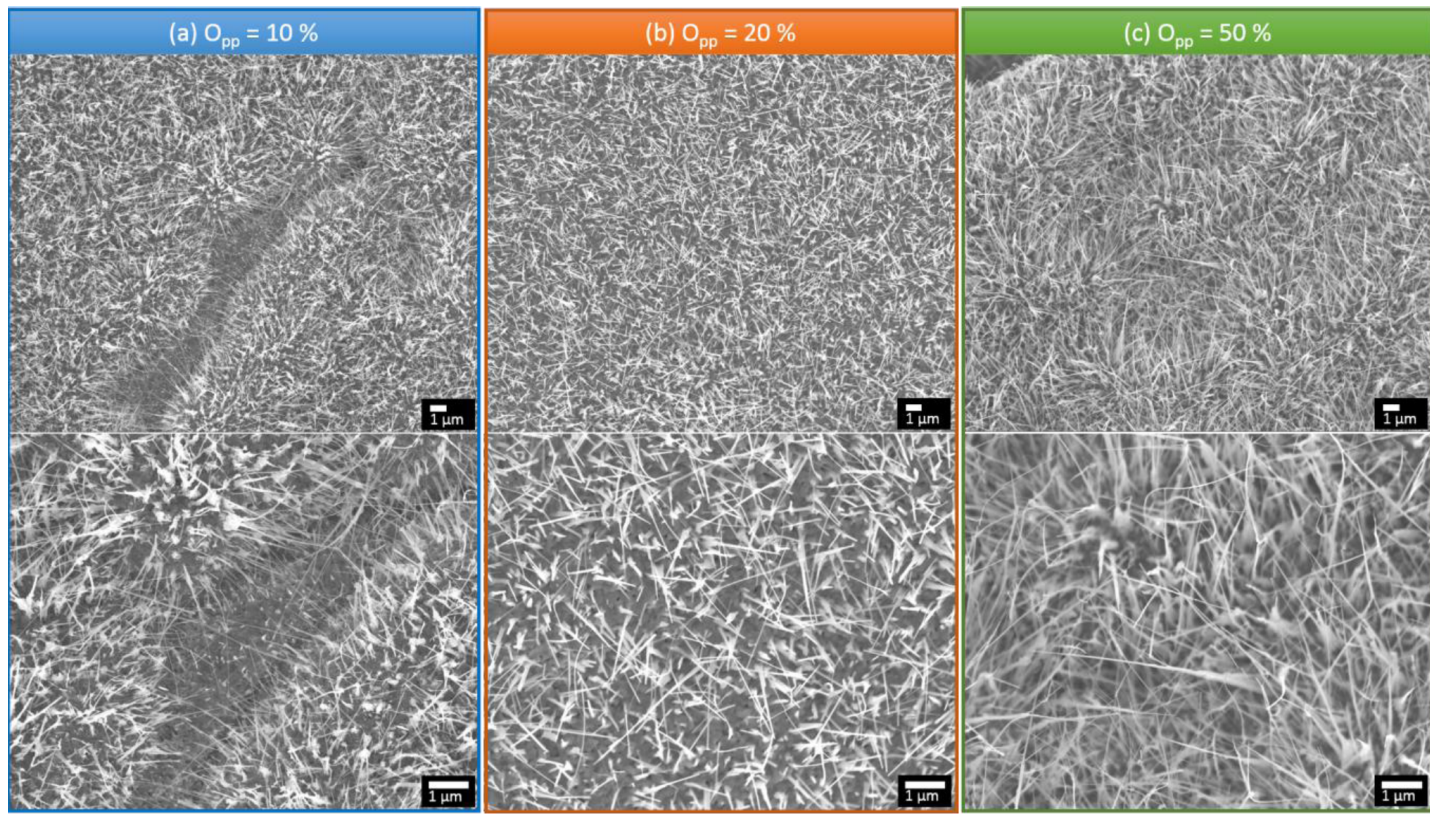

Figure 2. Influence of oxygen partial pressure on the growth of $\mathrm{CuO}$ nanowires. $(a): \mathrm{O}_{p p}=10 \%,(b): \mathrm{O}_{p p}=20 \%$, (c): $\mathrm{O}_{\mathrm{pp}}=50 \%$.

10 150 $\mathrm{nm}$ and $\geq 7 \mu \mathrm{m}$, respectively. In addition, the nanowires resembled a bamboo tree in which bunches of wires were formed simultaneously. Interestingly, the nanowires were grown uniformly on the entire foil surface. Therefore, a large number of wires could be extracted and obtained.

TEM, high-resolution TEM (HRTEM) and fast Fourier transform (FFT) images of the extracted nanowire are shown in Fig. 3. The single-crystal structure of the nanowire was detected in the HRTEM images (Fig. $3(\mathrm{~b}, \mathrm{c}))$, in which the interplanar spacing of the structure was $0.23 \mathrm{~nm}$, corresponding to the calculated spacing of the (200) plane of monoclinic $\mathrm{CuO}(\mathrm{a}=4.69 \AA, \mathrm{b}=3.42 \AA$, $c=5.13 \AA$ and $\beta=99.65^{\circ}$ ). Three bright dots representing $\mathrm{CuO}$ (200), (110) and (020) in the FFT dark-field image (Fig. 3(d)), as calculated from the HRTEM imagery (Fig. 3(c)) by Gatan Microscopy Suite (ver. 1.7.1), confirmed the $\mathrm{CuO}$ phase of the wire. Moreover, the data indicated that the growth of the nanowires was along the [100] direction.

The formation of the $\mathrm{CuO}$ nanowires can be summarized as follows: first, under a heat treatment,

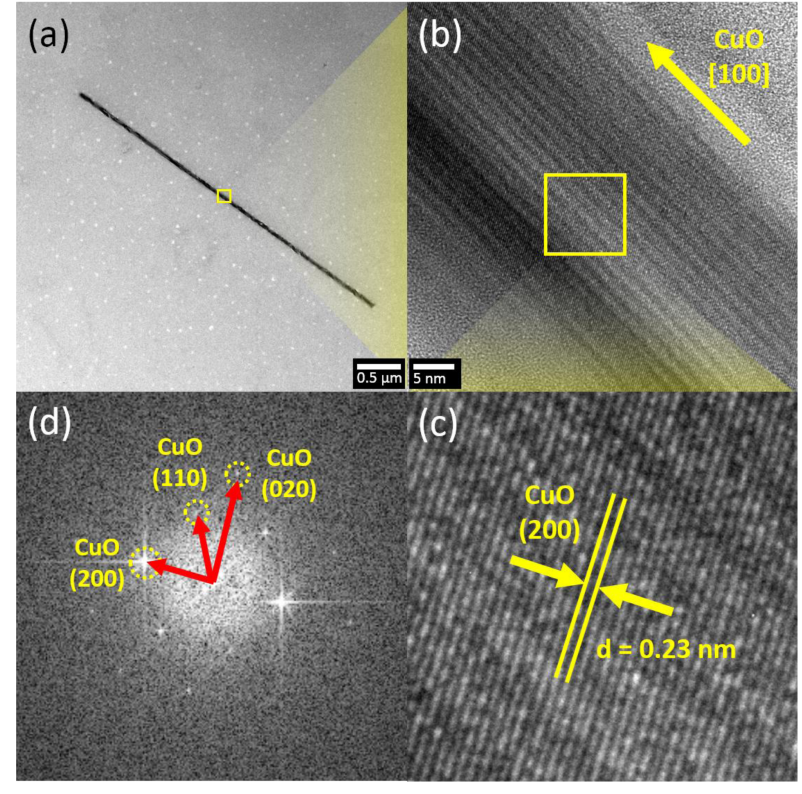

Figure 3. TEM (a), HRTEM (b, c) and FFT (d) images of the single $\mathrm{CuO}$ nanowire.

the atoms of $\mathrm{Cu}$ move and collide with each other randomly on the foil surface. By reacting with adsorbed oxygen from outside, an oxide layer is formed on the surface, which leads to the introduction of $\mathrm{CuO}$ nuclei and seeds. Second, the $\mathrm{CuO}$ seeds can easily become 
attractive sites for nearby atoms, molecules or nuclei. Thus, the seeds can be expanded in size or merged with several secondary seeds nearby. This explains why bunches of wires were formed. Third, crystallization was carried out with different priorities toward different faces depending on the preferred growth direction or preferred bonding direction.

\section{Field-effect properties of the device}

FESEM images of the single $\mathrm{CuO}$ nanowire devices are shown in Fig. 4. For a good device, only one wire was found to connect the two electrode terminals, and there were no impurities around the wire. Figs. 4(a) and 4(b) show devices with long and short single $\mathrm{CuO}$ nanowires, respectively. Occasionally, a short wire was found to have overlapped the main wire, as shown in Fig. 4(b). With regard to our observation, the probability of the appearance of a single $\mathrm{CuO}$ nanowire device relative to that of all other types was estimated to be around $25 \%$.

The current of the drain source ( $\mathrm{I}_{\mathrm{DS}}$ ) versus the gate $\left(V_{G}\right)$ and the drain-source $\left(V_{D S}\right)$ curves of a typical single $\mathrm{CuO}$ nanowire FET are shown in Fig. 5. In Fig. 5(b), the absolute conductance levels of the nanowire obtained under gate voltages $\left(\mathrm{V}_{\mathrm{G}}\right)$ of $-70,-60,-50,-40$, and $-30 \mathrm{~V}$ are shown to decrease monotonically as the gate potential is increased. This suggests that the device exhibited p-type semiconducting characteristics. This

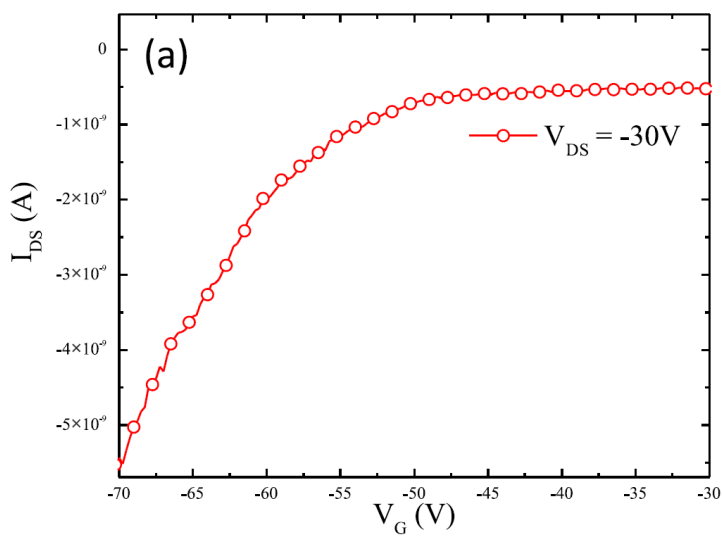

result is comparable to the findings of Liao et al. [4]. In our data, a high carrier concentration along with surface adsorption of the $\mathrm{CuO}$ nanowire may cause $\mathrm{I}_{\mathrm{DS}}$ to be unsaturated. Usually, nanowire FETs show field- effect characteristics which are weaker than those of thin films. Nevertheless, the successful operation of the device demonstrates the great potential of fabricating sensors and optical devices based on single-metal oxide nanowires.
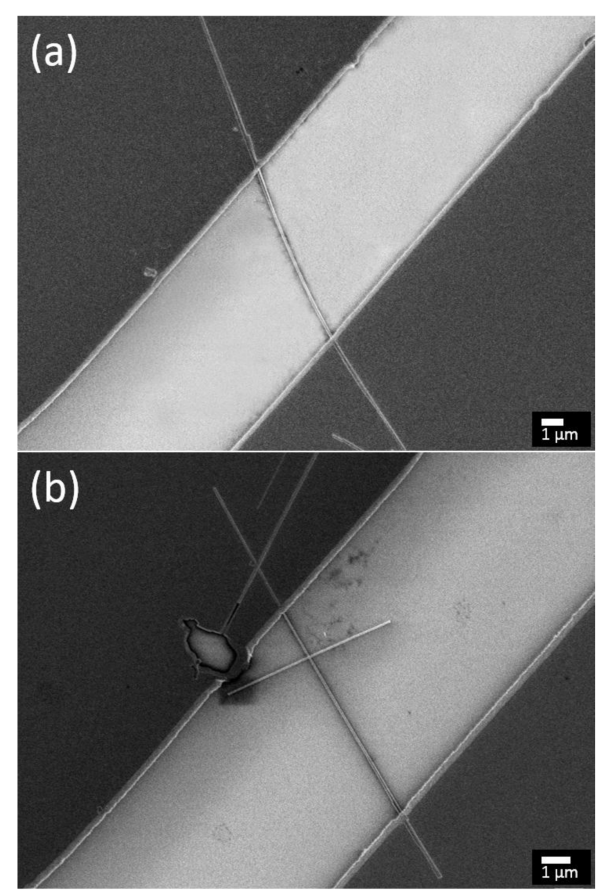

Figure 4. FESEM images of the single $\mathrm{CuO}$ nanowire devices.

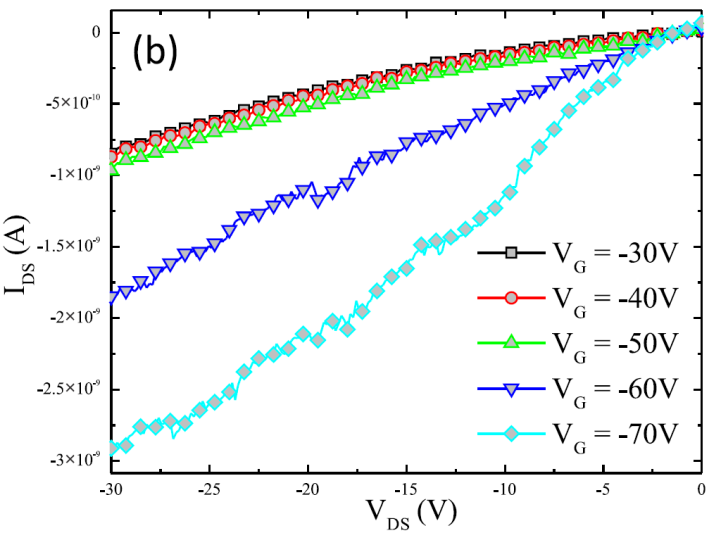

Figure 5. I-V characteristics of a single $\mathrm{CuO}$ nanowire FET. 


\section{Conclusion}

In conclusion, $\mathrm{CuO}$ nanowires with diameters of 10 150 $\mathrm{nm}$ and lengths of $\geq 7 \mu \mathrm{m}$ were synthesized by means of thermal oxidation. A feasible process of fabricating single-nanowire devices was introduced and used to make a single $\mathrm{CuO}$ nanowire field-effect transistor. The I-V characteristics of the devices confirm the $\mathrm{p}$-channel mode controlled by the gate and drain bias.

\section{Acknowledgement}

This work was supported by the Priority Research Centers Program, the Mid-career Researcher Program, and the Basic Science Research Program through a grant from the National Research Foundation of Korea (NRF) funded by the Korean government (MSIP) (No. 2009-0093819, 2011-0017245, and 2008-0062617). This research was also supported by the Kyungpook National University Fund of 2012.

\section{References}

[1] S. R. Gowda, A. L. M. Reddy, X. Zhan, and P. M. Ajayan, Nano Lett. 11, 3329 (2011).
[2] Y. Hu , J. Zhou , P.-H. Yeh , Z. Li , T.-Y. Wei, and Z. L. Wang, Adv. Mater. 22, 3327 (2010).

[3] F. Gu, L. Zhang, X. Yin, and L. Tong, Nano Lett. 8, 2757 (2008).

[4] L. Liao, Z. Zhang, B. Yan, Z. Zheng, Q. L. Bao, T. Wu, C. M. Li, Z. X. Shen, J. X. Zhang, H. Gong, J. C. Li, and T. Yu, Nanotechnology 20, 085203 (2009).

[5] I. Yavari, E. Sodagar, and M. Nematpour, Helv. Chim. Acta 97, 420 (2014).

[6] L. Ben-Xia, W. Yuan-Yuan, and W. Yan-Fen, Acta Phys. Chim. Sin. 25, 2366 (2009).

[7] A. Aslani, and V. Oroojpour, Physica B406, 144 (2011).

[8] S. Liu, J. Tian, L. Wang, X. Qin, Y. Zhang, Y. Luo, A.M. Asiri, A.O. Al-Youbi, and X. Sun, Catal. Sci. Technol. 2, 813 (2012).

[9] P. Poizot, S. Laruelle, S. Grugeon, L. Dupont, and J. M. Tarascon, Nature 407, 496 (2000).

[10] S. Manna, K. Das, and S. K. De, ACS Appl. Mater. Interfaces 2, 1536 (2010).

[11] X. Song, H. Yu, and S. Sun, J. Colloid Interface Sci. 289, 588 (2005).

[12] S. Liu, J. Tian, L. Wang, Y. Luo and X. Sun, Catal. Sci. Technol. 2, 339 (2012).

[13] X. Jiang, T. Herricks, and Y. Xia, Nano Lett. 2, 1333 (2002). 